\title{
Synthesis of Perfluoroalkyl Alcohol, 3-(Perfluorooctyl)propan-2-ol, Using Two-Step Alcoholization
}

\author{
Nguyen Trung Hieu, Beomseok Tae*, Junseo Park, and Kwang Nam Lee \\ Department of Chemical Engineering, Hankyong National University, Anseong-si, Gyeonggi-do 456-749, Korea. \\ ${ }^{*}$ E-mail: gaia@hknu.ac.kr
}

(Received August 8, 2012; Accepted September 10, 2012)

\begin{abstract}
Perfluorooctyl)propan-2-ol was synthesized using an alcoholization process. The synthesis consisted of two steps: (1) addition of the olefin propylene to perfluorooctyl iodide to yield the corresponding iodo-adduct perfluorooctyl propyl iodide and (2) hydrolysis of the adduct reacted with water and dimethylformamide to yield the final product. The adduct and product were analyzed using gas chromatography to determine the yield and purity. The optimal conditions for each step of the process were determined, and a 93\% yield of adduct and 95\% yield of final product was achieved.
\end{abstract}

Key words: Perfluoroalkyl alcohol, Perfluoroalkyl iodide, Alcoholization, Fluorinated surfactant

\section{INTRODUCTION}

Fluorinated surfactants are super surfactants that can decrease the surface tension of water below the lower limit reached by hydrocarbon-type surfactants. Since a perfluoroalkyl chain is not only hydrophobic but also oleophobic, fluorinated surfactants can serve as oil and fat repellents. Compounds consisting of a fluorinated chain and a hydrocarbon group can function as surfactants in hydrocarbon media. ${ }^{1-3}$ In perfluorinated surfactants, hydrogen in the hydrophobic segment is replaced by fluorine, while the hydrophobic section of partially fluorinated surfactants contains both fluorine and hydrogen atoms. ${ }^{1,4}$ Unlike hydrocarbon hydrophobe surfactants, partially fluorinated hydrophobes consist of two mutually phobic sections that are not compatible. Partially fluorinated surfactants therefore exhibit anomalies in macroscopic characteristics, such as the critical micelle concentration $(\mathrm{cmc})$, and also in microscopic phenomena. ${ }^{1}$

Direct fluorination with elemental fluorine is not practical for the commercial synthesis of fluorinated surfactants. ${ }^{1}$ Since the 1950 s, perfluoroalkyl groups have been synthesized from perfluoroalkyl iodide $\left(\mathrm{R}_{\mathrm{F}} \mathrm{I}\right)$ that is easily converted into $\mathrm{R}_{\mathrm{F}}$ radicals with a high reactivity towards unsaturated molecules. Further, $\mathrm{R}_{\mathrm{F}} \mathrm{I}$ can be used to make specific compounds with $\mathrm{R}_{\mathrm{F}}$ groups in many sizes and shapes, attached in precise positions in alkenes and alkynes of a large variety of structures (Scheme 1) $)^{5-8}$ There are a great number of useful products that are made using $\mathrm{R}_{\mathrm{F}} \mathrm{I}$ in this way, including herbicides, pharmaceuticals, surface treating compounds, polymers, and other important classes of products. ${ }^{5}$ However, $\mathrm{R}_{\mathrm{F}} \mathrm{I}$ does not react directly with nucleophiles such as $\mathrm{OH}^{-}$or $\mathrm{NH}_{3}$, it is therefore reacted with ethylene to form perfluoroalkylethyl iodide $\left(\mathrm{R}_{\mathrm{F}} \mathrm{CH}_{2} \mathrm{CH}_{2} \mathrm{I}\right)$ which can be readily converted to the corresponding alcohols, thiols, and sulfonyl chlorides that are used as intermediates in the synthesis of fluorinated surfactants. ${ }^{1,5,8-10}$

This method has been used to prepare several fluorinated compounds that have been reported in previous studies. ${ }^{5,10-15}$ A hydrolysis process in the presence of a catalyst can be used to transform (perfluoroalkyl)-iodoalkanes (for example, perfluoroalkyl ethyl iodide) into perfluoroalkyl alcohols. ${ }^{16,17}$ In addition, the perfluoroalkyl groupcontaining alcohols can be directly obtained. However, it is also possible to obtain the $\mathrm{R}_{\mathrm{F}} \mathrm{CH}_{2} \mathrm{CH}_{2} \mathrm{OCOR}$ esters first and to convert them then into the alcohols by using a hydrolysis reaction. The hydrolysis is easily performed by adding water in quantities $1-100$ times the molar amount of ester present at $80-160{ }^{\circ} \mathrm{C} .{ }^{9}$

In this article, an intermediate for fluorinated surfactants, named 3-(perfluorooctyl)propan-2-ol (PFAA), was synthe-

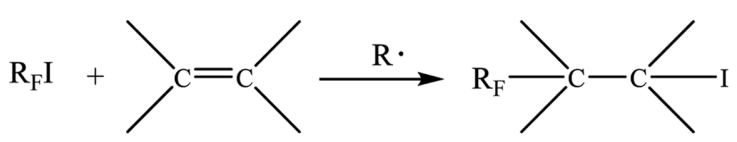

(Addition to Alkenes)

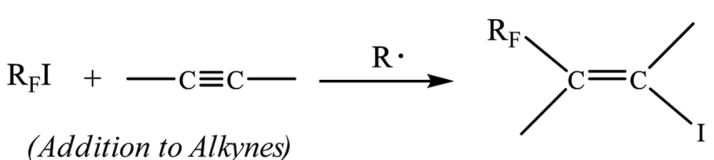

Scheme 1. Radical addition of $\mathrm{R}_{\mathrm{F}} \mathrm{I}$ to alkenes and alkynes. 

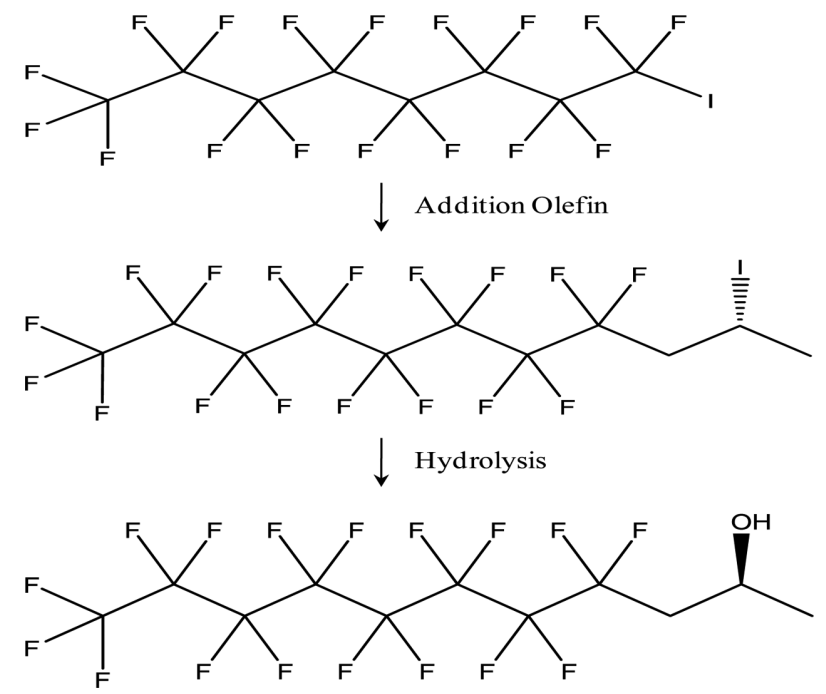

Scheme 2. Two step protocol for synthesizing 3-(perfluorooctyl)propan-2-ol.

sized from $\mathrm{R}_{\mathrm{F}} \mathrm{I}$, perfluorooctyl iodide (PFOI), via a twostep protocol consisting of propylene (PP) addition followed by hydrolysis (Scheme 2). In order to find the optimal conditions for the processes, the reactions were performed in a high-pressure reactor. The PFOI was added with PP gas, giving the adduct 3-(perfluorooctyl)-2-iodo-propane (PFAI) with $\sim 93 \%$ yield at the end of the first step. After the second step, $\sim 95 \%$ yield of PFAA was obtained from PFAI by hydrolysis. Moreover, fractional distillation at their boiling points increased the final product concentrations up to $99 \%$.

\section{RESULTS AND DISCUSSION}

In this article, the fluorinated compounds PFOI, PFAI, and PFAA were defined using gas chromatography (GC) analysis and Fourier transform infrared spectroscopy (FTIR). Table 1 shows the characteristic retention times for each compound based on the different components that enter the gas phase and are then swept towards the detector by a carried gas flow at different times. ${ }^{18-20}$ Furthermore, the compounds were distinguished based on functional groups, which was demonstrated by the FT-IR spectra shown in Fig. 1. A peak corresponding to the vibration of

Table 1. Gas chromatography spectra chemical shift values of fluorinated compounds

\begin{tabular}{cc}
\hline Fluorinated compounds & Retention time $(\mathrm{min})$ \\
\hline PFOI & 1.963 \\
PFAI & 3.297 \\
PFAA & 2.533 \\
\hline
\end{tabular}

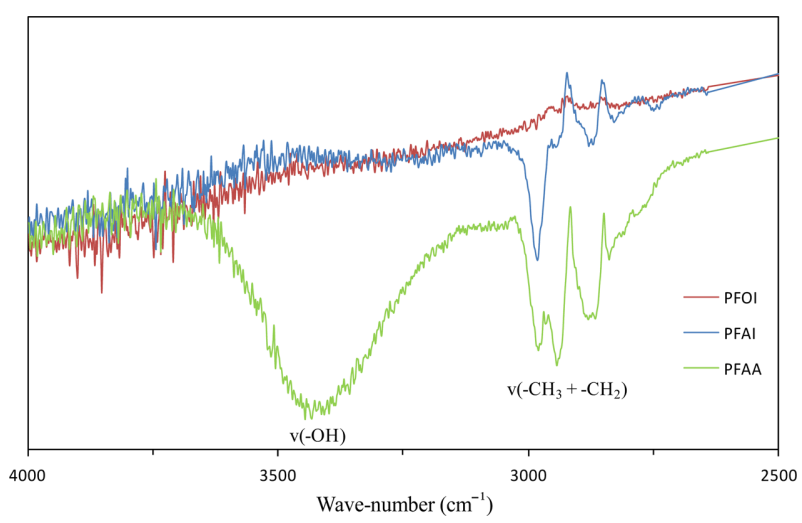

Fig. 1. FT-IR spectra of PFOI, adduct PFAI, and product PFAA.

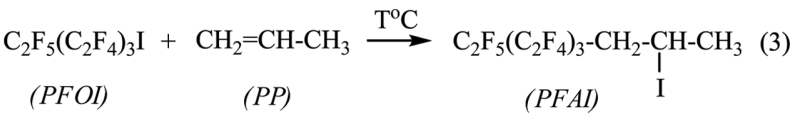

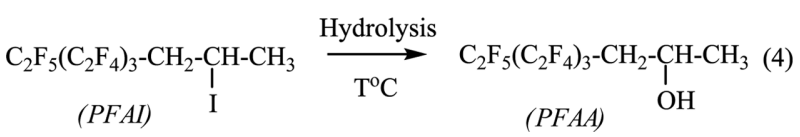

Scheme 3. Addition and hydrolysis reactions.

the hydroxyl group in PFAA can be observed at $3430 \mathrm{~cm}^{-1}$. At $2940 \mathrm{~cm}^{-1}$, the vibration of $\mathrm{C} \mathrm{sp}^{3}$, corresponding to the alkyl groups in the PFAI and PFAA, can be seen. The spectrum of PFOI does not indicate any signal in this zone, demonstrating that differences between the compounds can be distinguished clearly using this method.

The synthesis of PFAA was performed as reported in Scheme 2 via addition of PP to PFOI to give the corresponding iodo adduct, PFAI (Eq. (3)), followed by hydrolysis of the obtained PFAI with water in the presence of dimethylformamide (DMF) (Eq. (4)) (Scheme 3).

\section{The Addition Reaction}

The PP gas was added to PFOI with different volumes controlled by flow rate, for different reaction times, and at different temperatures. The amount of reacted PP in the reaction chamber was determined over the formed PFAI, which was measured by GC analysis. Fig. 2 shows the effective PP gas that reacted with PFOI at $150{ }^{\circ} \mathrm{C}$ over time with a variety of PP flow rates. At the low PP flow rate of $1 \mathrm{sccm}$ (standard cubic centimeters per minute), almost all of the PP gas fed into reactor was absorbed completely in the PFOI volume giving the PFAI adduct. The absorbance decreased when the flow rate was increased up to 3,5 , and $30 \mathrm{sccm}$; this suggested that the reaction is dominant in the first-order where the reaction molar ratio of PP to PFOI is one. Therefore, when the amount of fed 


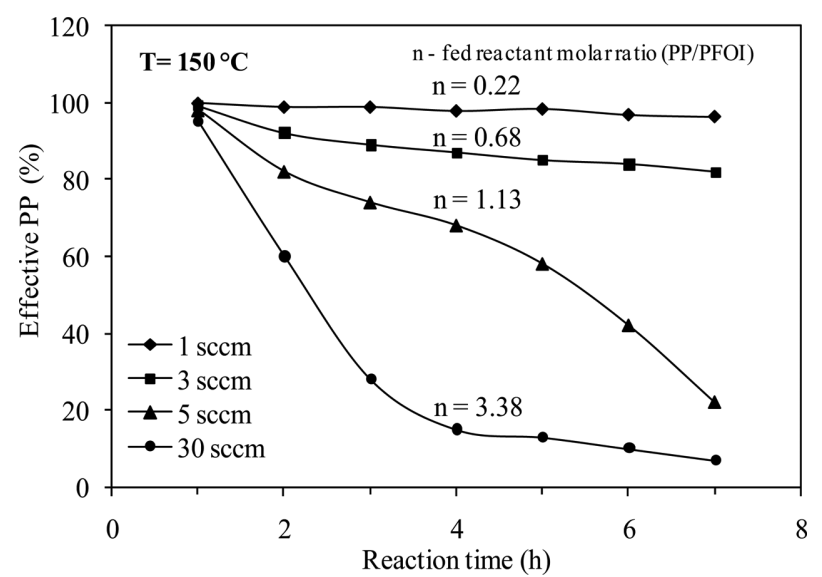

Fig. 2. Plot of the relationship between effective PP percentage and reaction time at $150{ }^{\circ} \mathrm{C}$ and different PP flow rates.

PP was higher than that at the saturation level, the absorbance was reduced. In order to determine the saturation point, the fed reactant molar ratio (n) was defined by division of the total moles of PP by the starting moles of PFOI, where the total moles of PP were calculated using an equation of state of an ideal gas based on the flow rate of PP. For example, after $4 \mathrm{~h}$ of reaction, the fed reactant molar ratios were 0.22 for the $1 \mathrm{sccm}$ flow rate and 0.68 for the $3 \mathrm{sccm}$ flow rate, which are much lower than 1 . The fed PP was being absorbed, meaning that the reaction was occurring. In the case of the $5 \mathrm{sccm}$ flow rate, the effective PP percentage decreased rapidly as $n$ was 1.13 . The absorbance can be seen to be extremely low for the $30 \mathrm{sccm}$ flow rate, and it reaches $18 \%$ at $4 \mathrm{~h}$, which corresponds to an $n$ value of 3.38 .

The PP flow rate-controlled reaction enables control over the reaction time required to achieve a high yield of the PFAI adduct. Fig. 3 shows the relationship between

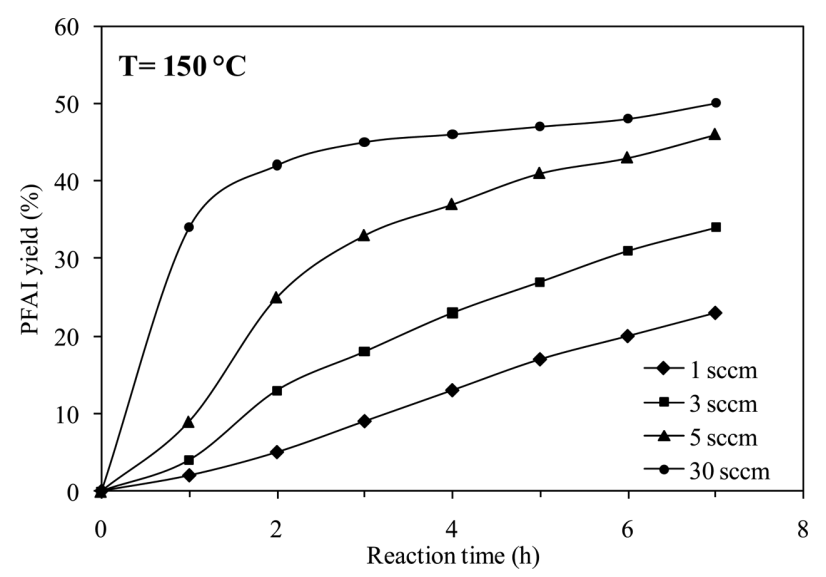

Fig. 3. Plot of the formed PFAI depending on reaction time at $150{ }^{\circ} \mathrm{C}$ and different PP flow rates.

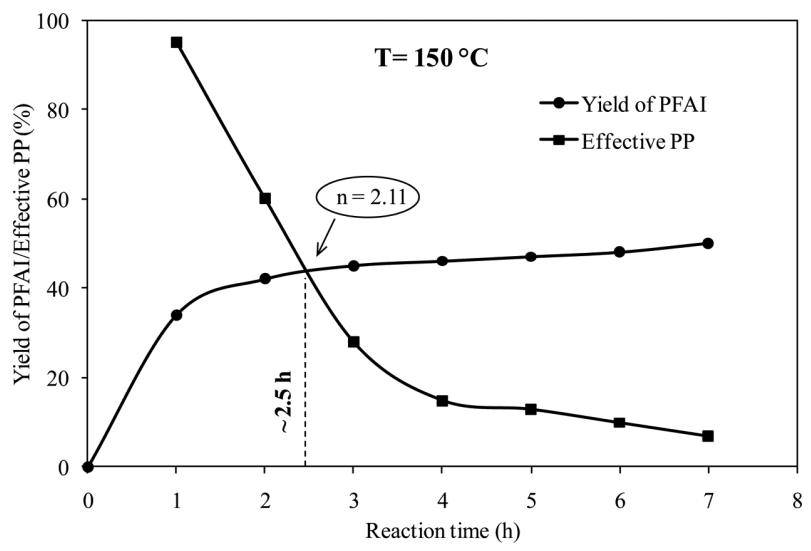

Fig. 4. Determination of the optimal reaction conditions of PP flow rate and time at $150{ }^{\circ} \mathrm{C}$.

formed PFAI and reaction time at $150{ }^{\circ} \mathrm{C}$ and different PP flow rates. It is clear that in the zone of pre-saturation, the more PP is fed the higher the amount of PFAI is formed. The $30 \mathrm{sccm}$ flow rate enabled more than $40 \%$ yield to be achieved in just $2 \mathrm{~h}$, which was considerably higher than the yield for the lower flow rates. However, after this time, only small further increases in yield can be seen for the 30 sccm flow rate, which is highly inefficient in terms of the used PP reactant and time. In order to accommodate these factors and save materials, time, and energy, the effective PP percentage, PFAI yield, and reaction time were optimized (Fig. 4). The intersection between the effective PP plot and PFAI yield plot at $30 \mathrm{sccm}$ flow rate and $150{ }^{\circ} \mathrm{C}$ can be considered to be the point corresponding to the most efficient reaction conditions, where the PP and the PFOI are used with a molar ratio $\mathrm{n}=2$ for reaction time of approximately $2.5 \mathrm{~h}$. Although as mentioned, this is a first-order reaction, a value of $\mathrm{n} \sim 2$ is acceptable. This is because the reaction between the PP gas and the PFOI liquid is heterogeneous, and therefore, the absorbance of PP in PFOI also depends on the feeding method, which in this study, was bubbling.

Based on the obtained reaction conditions, the production of PFAI was investigated at different temperatures. Fig. 5 shows the plots of PFAI yield depending on temperature for reactions that were carried out for $2.5 \mathrm{~h}$ with $\mathrm{n}=1.0$ and $\mathrm{n}=2.0$. Both reactions gave the highest yields of PFAI at $270{ }^{\circ} \mathrm{C}, 70 \%$ for $n=1.0$ and $93 \%$ for $n=2.0$. These results suggested that this temperature, together with the reaction time $(2.5 \mathrm{~h})$ and the fed reactant molar ratio $(\mathrm{n}=2.0)$, are the best reaction conditions for synthesizing PFAI in a high yield. 


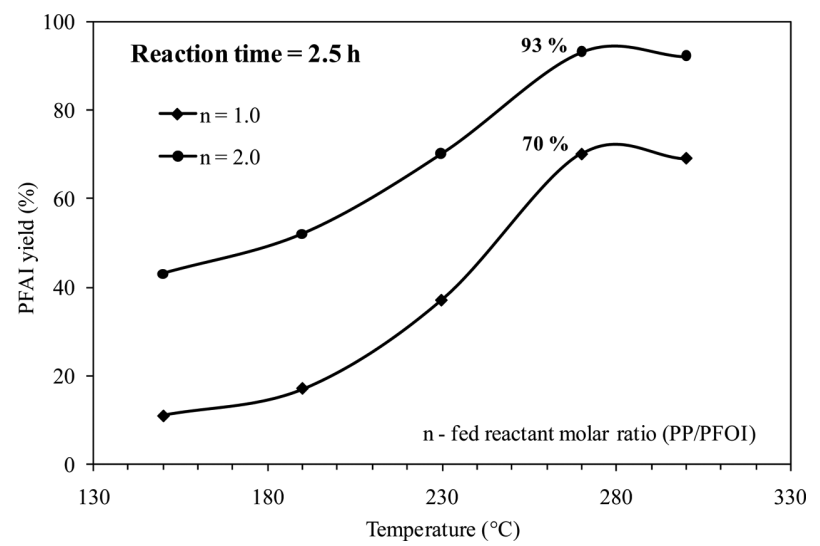

Fig. 5. Plot of the formed PFAI depending on temperature for $2.5 \mathrm{~h}$.

\section{The Hydrolysis Reaction}

PFAA was prepared from the obtained PFAI using a hydrolysis process, as seen in Eq. (4). In this work, DMF was used as an amide, containing a hydrogen atom and two lower methyl groups owing to its availability and its reactivity. ${ }^{9}$ The synthesis of the PFAA is represented by equations (5) and (6) (Scheme 4), where $\mathrm{R}_{\mathrm{F}}$ represents the perfluorooctyl group $\left(\mathrm{C}_{8} \mathrm{~F}_{17-}\right)$.

According to Hayashi, ${ }^{9}$ mechanistically, the perfluoroalkyl ester is produced from the halide, water, and the amide, and that total or partial conversion of the ester to the corresponding alcohol by hydrolysis is determined by the amount of water present in the reaction system. Thus, by controlling the amount of water relative to PFAI, it is possible to obtain the ester or the PFAA as the main product. For the hydrolysis reaction carried out in this work, the molar ratio of water to PFAI was at least $2: 1$, and that of DMF to PFAI was at least 5:1 (Fig. 6). If a small amount of water is used, the conversion of the starting PFAI is very low. In contrast, when the amount of water used is too high, the selectivity of the desired PFAA product is low. Further, the DMF is used as a reaction medium that can be used in large amounts. However, there is no advantage in using larger quantities of the amide, chiefly because of economic reasons involving the reactors and recovery of the amide. On the other hand, there is no

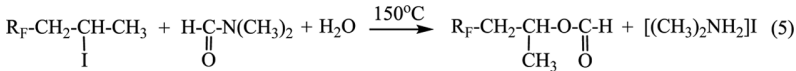

$$
\begin{aligned}
& \text { (PFAI) (DMF) (ester) }
\end{aligned}
$$

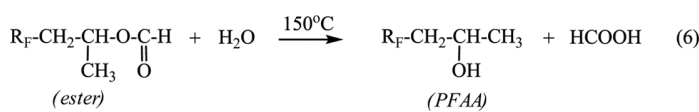

Scheme 4. PFAA synthesizing reactions.

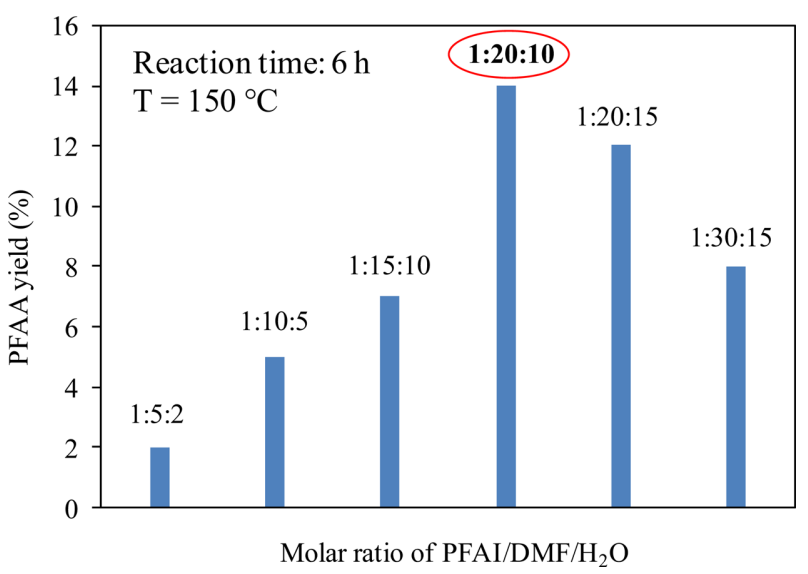

Fig. 6. Yield of PFAA depending on molar ratio of PFAI/DMF/ $\mathrm{H}_{2} \mathrm{O}$ at $150{ }^{\circ} \mathrm{C}$ for $6 \mathrm{~h}$.

advantage in using a low amount of amide as the smoothness of the reaction is decreased, and the conversion of the PFAI and the selectivity for the PFAA are very low. ${ }^{9}$ Accordingly, it is preferable to set the molar ratio of PFAI/ $\mathrm{DMF} / \mathrm{H}_{2} \mathrm{O}$ to $1: 20: 10$.

In this study, the reaction was performed at $150{ }^{\circ} \mathrm{C}$. If the reaction temperature is too high, the yield of undesirable side products increases, whereas if the reaction temperature is too low, the reaction velocity becomes slow, resulting in a decreased yield of product. At the selected reaction temperature, the yield of PFAA was investigated at different reaction times in the range of 3 to $12 \mathrm{~h}$ as shown in Fig. 7. The optimal reaction time when the reaction is complete with a $95 \%$ yield of PFAA was found to be $12 \mathrm{~h}$. Moreover, the yield of PFAA could be enhanced by fractional distillation, which thoroughly separates the product of the hydrolysis reaction from the unreacted materials and by-products, including $\mathrm{R}_{\mathrm{F}} \mathrm{CH}=\mathrm{CH}-\mathrm{CH}_{3},\left(\mathrm{CH}_{3}\right)_{2} \mathrm{NH}_{2} \mathrm{I}$,

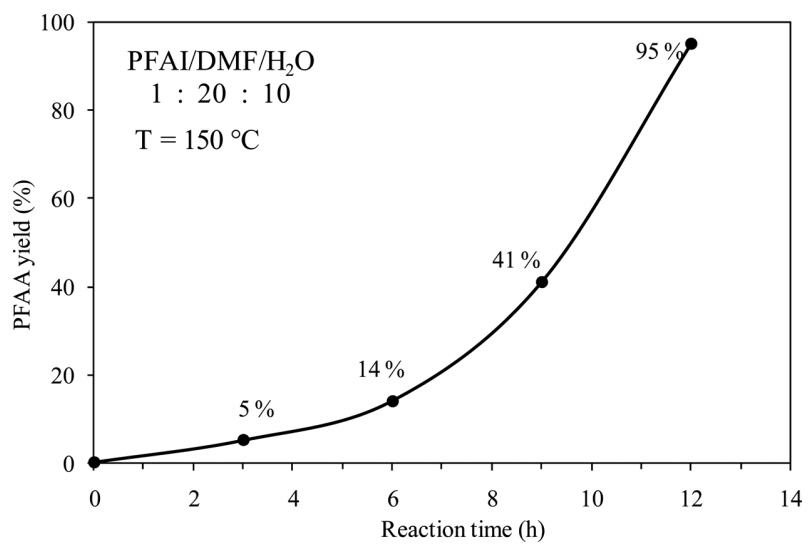

Fig. 7. Yield of PFAA depending on reaction time at $150{ }^{\circ} \mathrm{C}$ with molar ratio of $\mathrm{PFAI} / \mathrm{DMF} / \mathrm{H}_{2} \mathrm{O}=1: 20: 10$. 
and $\mathrm{HCOOH}$. In this study, fractional distillation was performed at $110^{\circ} \mathrm{C}$, which is the boiling point of the PFAA, and the concentration of PFAA was increased to $99 \%$. Thus, by using the hydrolysis, a high yield of the PFAA could be obtained from the ester that was a product of the PFAI and the DMF. The high yield of PFAA achieved after the long reaction time demonstrated that it is only necessary to perform the hydrolysis reaction such that close contact between the reagents is maintained. This was suitably accomplished by stirring during the period of the reaction.

\section{CONCLUSION}

The fluorinated compound, 3-(perfluorooctyl)propan-2-ol, is a useful intermediate for producing fluorinated surfactants. In this study, two-step alcoholization involving an addition reaction followed by hydrolysis was used to synthesize it from perfluorooctyl iodide. The optimal reaction conditions were investigated, and high yields of $93 \%$ for the PFAI adduct and $95 \%$ for the PFAA product were achieved. Furthermore, an appropriate fractional distillation at their boiling points enabled concentrations of $99 \%$ purity to be obtained. This method could be applied to the synthesis of a wide variety of useful fluorinated compounds.

\section{EXPERIMENTAL}

Gas chromatography analyses were performed on an HP-5890 series II instrument using a fused silica capillary HP-5 column $(30 \mathrm{~m} \times 0.32 \mathrm{~mm})$. Typical operating conditions were as follows: temperature program, $200^{\circ} \mathrm{C}$ for $2 \mathrm{~min}$, followed by increase to $250^{\circ} \mathrm{C}$ at the rate of $40^{\circ} \mathrm{C} /$ min; carrier gas, He (flow rate: $1 \mathrm{~mL} / \mathrm{min}$ ). FTIR spectra were measured using a Jasco FT/IR-6100 instrument. PFOI (Daikin) and commercial PP gas contained in a tank were used for the addition reaction. DMF (Junsei) was used for the hydrolysis reaction. These reactions were operated in a high-pressure stainless steel reactor that was heated by a jacket controlled by a heating controller. The PP gas was injected into the reactor through a mass flow meter (Mykrolis FC-2805) connected to a digital flow controller (DFC4000 series of SMTEC company).

\section{Addition Reaction}

\section{Propylene flow rate-controlled reaction}

$50 \mathrm{~g}(0.092 \mathrm{~mol})$ of PFOI was added to a $360 \mathrm{~mL}$ stainless steel reactor under stirring. This was heated at the rate of $10{ }^{\circ} \mathrm{C} / \mathrm{min}$ to $150{ }^{\circ} \mathrm{C}$. When this temperature was reached, the PP gas was injected as bubbles into the volume of the PFOI, at flow rates of $1,3,5$ or $30 \mathrm{sccm}$ used depending on the specific experiment. The reaction pressure was not critical. During the reaction time, small amounts of sample were taken each hour for GC analysis.

\section{Temperature-controlled reaction}

PFOI (1.343 g, $\left.2.5 \times 10^{-3} \mathrm{~mol}\right)$ and PP gas $\left(2.5 \times 10^{-3}\right.$ mol for $\mathrm{n}=1.0,5.0 \times 10^{-3} \mathrm{~mol}$ for $\mathrm{n}=2.0$ ) were added to $\mathrm{a}$ closed $20 \mathrm{~mL}$ stainless steel reactor under stirring for $2.5 \mathrm{~h}$ at $150,190,230,270$, or $300^{\circ} \mathrm{C}$ corresponding to different experiments. The reaction pressure was not critical. At the end of the reaction, samples were taken for GC analysis. The reaction that gave the highest yield of the product, 93\% PFAI, was repeated using larger amounts of chemicals. The PFAI concentration was increased to approximately $99 \%$ by fractional distillation at $150{ }^{\circ} \mathrm{C}$.

\section{Hydrolysis Reaction \\ Molar ratio-controlled reaction}

Using $2.940 \mathrm{~g}\left(5.0 \times 10^{-3} \mathrm{~mol}\right)$ of the $99 \%$ pure PFAI obtained in the previous reaction, mixture of DMF and $\mathrm{H}_{2} \mathrm{O}$ with molar ratios of PFAI/DMF/ $\mathrm{H}_{2} \mathrm{O}$ of 1:5:2, 1:10:5, $1: 15: 10,1: 20: 10,1: 20: 15$, and 1:30:15 were prepared. These reactions were carried out in a closed $20 \mathrm{~mL}$ stainless steel reactor under stirring for $6 \mathrm{~h}$ at $150{ }^{\circ} \mathrm{C}$. The reaction pressure was not critical. At the end of the reactions, the PFAA yield was analyzed using GC.

\section{Time-controlled reaction}

$50 \mathrm{~g}(0.085 \mathrm{~mol})$ of the $99 \%$ PFAI, $124 \mathrm{~g}$ of DMF, and $15.3 \mathrm{~g}$ of $\mathrm{H}_{2} \mathrm{O}$ corresponded to the optimum molar ratio of PFAI/DMF/ $/ \mathrm{H}_{2} \mathrm{O}$ of 1:20:10. The reaction was carried out in a $360 \mathrm{~mL}$ stainless steel reactor under stirring at $150{ }^{\circ} \mathrm{C}$ for $12 \mathrm{~h}$. The reaction pressure was not critical. During the reaction time, small amounts of sample were taken every $3 \mathrm{~h}$ for $\mathrm{GC}$ analysis.

The final product was separated from the reaction medium containing water and DMF using an extraction. That was based on different densities of components which are $1 \mathrm{~g} / \mathrm{mL}$ for water, $\sim 0.95 \mathrm{~g} / \mathrm{mL}$ for DMF, and $\sim 1.6 \mathrm{~g} / \mathrm{mL}$ for PFAA.

\section{REFERENCES}

1. Kissa, E. In Fluorinated Surfactants and Repellents; Marcel Dekker, Inc.: New York, 2001; Vol. 97, p 1.

2. Lehmler, H. J. Chemosphere 2005, 58, 1471.

3. Loganathan, B. G.; Sajwan, K. S.; Sinclair, E.; Senthil Kumar, K.; Kannan, K. Water Res. 2007, 41, 4611.

4. Frömel, T.; Knepper, T. P. In Reviews of Environmental Contamination and Toxicology: Perfluorinated Alkylated 
Substances; Whitacre, D. M.; Voogt, P. d., Ed.; Springer: New York, 2010; Vol. 208, p 161.

5. Brace, N. O. J. Fluorine Chem. 1999, 93, 1.

6. Petrov, V. A.; Krespan, C. G. J. Fluorine Chem. 2000, 102, 199.

7. Lewandowski, G.; Meissner, E.; Milchert, E. J. Fluorine Chem. 2006, 127, 337.

8. Davis, C. R.; Burton, D. J.; Yang, Z.-Y. J. Fluorine Chem. 1995, 70, 135.

9. Hayashi, T.; Matsuo, M. U.S. Patent 4001309 (to Asahi Glass Company, Ltd., Tokyo, Japan), 1977.

10. Brace, N. O. J. Fluorine Chem. 2005, 126, 7.

11. Brace, N. O. J. Fluorine Chem. 1999, 96, 101.

12. Brace, N. O. J. Fluorine Chem. 2001, 108, 147.

13. Gambaretto, G.; Conte, L.; Fornasieri, G.; Zarantonello, C.; Tonei, D.; Sassi, A.; Bertani, R. J. Fluorine Chem. 2003, 121, 57 .
14. Paterová, J.; Skalický, M.; Rybácková, M.; Kvícalová, M.; Cvacka, J.; Kvícala, J. J. Fluorine Chem. 2010, 131, 1338.

15. Yoneda, N. J. Fluorine Chem. 2004, 125, 7.

16. Bargigia, G.; Tonelli, C.; Tortelli, V. U.S. Patent 4748282 (to Ausimont S.p.A., Milan, Italy), 1988.

17. Day, R. I. U.S. Patent 3283012 (to E.I. Du Pont de Nemours), 1966.

18. McNair, H. M.; Miller, J. M. In Basic Gas Chromatography; John Wiley \& Sons, Inc.: New York, 1997; p 1.

19. Stuart, B.; Prichard, E. In Practical Laboratory Skills Training Guides; LGC, Teddington, UK: Cambridge, 2003; $\mathrm{p} 1$.

20. Grob, R. L.; Barry, E. F. In Modern Practice of Gas Chromatography; John Wiley \& Sons, Inc.: New Jersey, 2004; p 25. 